\title{
Survival after lumpectomy and mastectomy for early stage invasive breast cancer: the effect of age and hormone receptor status
}

\author{
E. Shelley Hwang, MD, MPH ${ }^{*}, 1$, Daphne Y. Lichtensztajn, MS $^{3}$, Scarlett Lin Gomez, PhD $^{3}$, \\ Barbara Fowble, MD², and Christina A. Clarke, PhD $^{3}$ \\ ${ }^{1}$ Duke University Comprehensive Cancer Center, Department of Surgery \\ 2University of California San Francisco Helen Diller Family Comprehensive Cancer Center, \\ Department of Radiation Oncology \\ ${ }^{3}$ Cancer Prevention Institute of California (CPIC)
}

\section{Abstract}

\begin{abstract}
Background-Randomized clinical trials (RCT) have demonstrated equivalent survival for breast conserving therapy with radiation (BCT) and mastectomy for early stage breast cancer. We studied a large, population-based series of women who underwent BCT or M to observe whether outcomes of RCT were achieved in the general population, and whether survival differed by surgery type when stratified by age and hormone receptor (HR) status.
\end{abstract}

\begin{abstract}
Methods-We obtained information regarding all women diagnosed in the state of California with stage I or II breast cancer between 1990 and 2004, treated with either BCT or mastectomy and followed for vital status through December 2009. We used Cox proportional hazards modeling to compare overall survival (OS) and disease-specific survival (DSS) between BCT and mastectomy groups. Analyses were stratified by age group $(<50$ years and $\geq 50$ years $)$ and tumor hormone receptor (HR) status.
\end{abstract}

Results-112,154 women fulfilled eligibility criteria. Women undergoing BCT had improved OS and DSS when compared to women with mastectomy (adjusted HR for OS entire cohort 0.81, 95\% CI $0.80-0.83)$. The DSS benefit with BCT compared to mastectomy was greater among women age 250 with HR-positive disease (HR $0.86,95 \%$ CI $0.82-0.91$ ) than among women age $<50$ with HR-negative disease (HR 0.88, 95\% CI 0.79-0.98); however, this trend was seen among all subgroups analyzed.

Conclusion-Among patients with early stage breast cancer, BCT was associated with improved DSS. These data provide confidence that BCT remains an effective alternative to mastectomy for early stage disease regardless of age or HR status.

\section{BACKGROUND}

For over two decades, it has been believed that early stage breast cancer outcomes were equivalent with either mastectomy or breast conserving surgery with adjuvant radiation (breast conserving therapy: BCT). Accordingly, BCT rates have increased throughout the United States ${ }^{1,2}$. The proportion of women choosing BCT has also increased among breast

\footnotetext{
*Corresponding author: 465 Seeley Mudd Building, Duke University Medical Center, Durham ND, 27710; office: (919) 684-6849. Presented in abstract form at the San Antonio Breast Cancer Symposium, December 2010

Financial Disclosures: none
} 
cancer patients of all ages ${ }^{3}$ and racial/ethnic groups, although important disparities still persist 4,5 .

However, recent reports have suggested a rising use of mastectomy in more affluent regions ${ }^{6}$, in those undergoing $\mathrm{MRI}^{7,8}{ }^{8}$, in younger women ${ }^{6}$, and among those with in situ disease ${ }^{6}$. Although the reasons contributing to this observed trend towards mastectomy remain largely speculative, they may relate to improvements in reconstruction techniques, or to women's attitudes towards mastectomy, including the desire to reduce anxiety associated with long-term surveillance. In addition, there has been renewed interest in mastectomy based on recent studies reporting higher recurrence rates among BCT recipients with tumor characteristics suggesting increased locoregional recurrence risk 9,10 . In light of the reports showing renewed interest in mastectomy, and because there have been few opportunities to observe long-term treatment-associated outcomes in the general population, we asked whether the comparable survival outcome of BCT compared to mastectomy as seen in randomized controlled trials could be generalized to the non-clinical trial population. We used the large, population-based prospective California Cancer Registry (CCR) to explore observational associations between surgery type, overall survival (OS) and breast cancerspecific survival among women diagnosed with stage I-II breast cancer to determine how the choice of mastectomy might impact long-term outcomes for early stage disease, and importantly, whether these results persisted despite age at diagnosis or ER status.

\section{METHODS}

\section{Case Ascertainment and Data Collection}

Cases eligible for this study were all female residents of California newly diagnosed with invasive breast cancer (International Classification of Diseases--Oncology, 3rd edition (ICD-O-3) morphology codes C50.0-50.9 and reported to the state-mandated California Cancer Registry (CCR) with a new, unilateral T1/T2 stage I or II disease diagnosed during the period January 1, 1990 through December 31, 2004. Eligible cases were treated with either definitive BCT defined by receipt of both lumpectomy and radiation, or mastectomy without radiation and had at least 5 years of follow up. Patients treated with either lumpectomy without radiation or mastectomy with radiation were excluded. Vital status and underlying cause of death if applicable, routinely determined by the CCR through hospital follow-up and routine linkage with state and national death indices, were collected up to December 31, 2009. 215,049 women aged 18-80 diagnosed with unilateral breast cancer between 1990 and 2004 were identified. Cases were excluded sequentially as follows: 29,713 for a diagnosis of a previous primary cancer; 36,115 for treatment with lumpectomy without adjuvant radiation $(23,617)$ or mastectomy with adjuvant radiation $(11,002)$ or no/ unknown/other treatment (1496); 30,870 women for unknown HR status; 4,074 for tumor size missing or $>5 \mathrm{~cm}$; and 2,123 for date of surgery more than 120 days after the initial date of diagnosis, based on the concern that these cases may have been treated with neoadjuvant chemotherapy. Node status was treated as a categorical variable according to proportion of positive nodes, as current nodal staging of $\mathrm{N} 2$ and $\mathrm{N} 3$ disease includes internal mammary node data and this information was not reliably collected by the registry. The final cohort fulfilling all eligibility criteria consisted of 112,514 women.

\section{Tumor subtype information}

The CCR has collected information on ER and PR expression of breast cancers since 1990, the first year of cohort for this study. Each marker was classified as positive (at least 5\% nuclear staining), negative, borderline, not tested, not recorded, or unknown on the basis of pathology or medical record information. Cases were deemed HR-positive if tumors expressed ER, PR, or both. If both receptors were negative, cases were classified as HR 
negative. Patients whose receptor status was borderline, not tested, or not recorded were excluded. HER2 receptor testing results were not available uniformly for years prior to 2006, and were therefore not included in the analysis.

\section{Neighborhood socioeconomic information}

We used a measure of neighborhood-level SES assigned to patients' residential address at diagnosis, based on a previously described principal components-derived index that incorporates census-block group level Census 1990 or 2000 data on education, income, occupation, and housing costs, among others ${ }^{11}$. Each case was assigned a neighborhood SES quintile based on the distribution of the composite SES index across the state of California, with 1 representing the lowest SES quintile.

\section{Statistical Analysis}

Overall survival between surgery groups was compared using a Kaplan-Meier estimator. Outcome between surgery groups was compared using the log rank comparison. Cumulative incidence functions were used to estimate the probability of 5-year breast cancer-specific survival in the presence of competing risks. Adjusted Cox proportional hazards models were used to estimate hazard ratios (HR) for DSS or OS between BCT and mastectomy groups; the full models included surgery type, tumor grade, tumor size (continuous variable), proportion of positive lymph nodes, age at diagnosis, year of diagnosis, SES, and race. For cause-specific survival, cases that were alive or had died of other causes were censored at time of last follow up or death. Separate analyses were conducted for each combination of hormone receptor status and age group $<50$ vs $\geq 50$ based on age at diagnosis. To test the proportionality assumption we graphed the $\log (-\log )$ of survival versus the $\log$ of time. No violations were found. Competing risk analyses were conducted using the cmprsk package implemented in R (R Foundation for Statistical Computing, Vienna, Austria). All other statistical analyses were conducted in SAS version 9.2 (SAS Institute, Cary, NC).

\section{RESULTS}

The cohort of 112,154 eligible women included 61,771 (55\%) who received lumpectomy and radiation and 50,383 (45\%) who had mastectomy without radiation. Median follow up time was 110.6 months.

\section{Distributions of Patient/Tumor Factors by Surgery Type}

Table 1 shows demographic and clinical characteristics of the patient cohort. $25 \%$ of the BCT group and $26 \%$ of the mastectomy group were younger than 50 years of age at diagnosis; only $6.0 \%$ of the cohort overall were younger than 40 years, and $23 \%$ were between 70 and 80 years at diagnosis. Nearly a quarter of the cohort was non-white, including $11.5 \%$ Hispanic, $8.6 \%$ Asian/Pacific Islander, and 5.0\% black.

Surgical practice patterns for early stage breast cancers differed by year of diagnosis between 1990 and 2004. In the overall cohort, the proportion of patients treated with BCT increased from $37 \%$ between $1990-92$ to $62 \%$ between 2002-04. The proportion of women undergoing lumpectomy was nonlinear with respect to decade of age at diagnosis; $51 \%$ of women under the age of 40 underwent BCT compared with 59\% of women age 50-59 and $51 \%$ of women age 70-80. Non-Hispanic whites had the highest rate of BCT (57\%) followed by non-Hispanic blacks, Hispanics, and non-Hispanic Asians/Pacific Islanders (55\%, 51\% and $45 \%$, respectively). The mastectomy rate declined among all racial groups during the study period. 
Median tumor size was $1.5 \mathrm{~cm}$, with larger average tumor size seen among the mastectomy group, as expected. The rate of BCT among tumor size categories was compared among age groups $(<40,40-54,55-69,70-80)$ and decreased with tumor size across all age groups (Figure 1). Interestingly, the use of BCT varied by age even among tumors $\leq 2 \mathrm{~cm}$ where the youngest and oldest age groups had the lowest BCT rate. In larger tumors $(>2 \mathrm{~cm}) \mathrm{BCT}$ rate declined by age (Figure 1).

Differences in surgery type were seen among tumor histologies. Lobular cancers were associated with a higher mastectomy rate when compared to ductal tumors (53\% compared to $44 \%$, respectively), a result that was seen even when limiting the analysis to T1 lesions only (44\% compared to 38\%). A greater proportion of grade I tumors were more likely to be treated with BCT than non-low grade tumors. Patients with grade III and HR-negative tumors were more likely to undergo mastectomy than the overall population $(50 \%$ in both groups).

\section{Impact of Surgery Type on Survival Endpoints}

A total of 31,416 deaths were identified in the cohort during the study period, including $39 \%$ due to breast cancer as the underlying cause. 5-year OS in the group was $89.3 \%$ (95\% CI 89.2-89.5\%); DSS was $94.4 \%$ (95\% CI 94.2-94. 5\%)

Cox multivariable analysis was performed to compare OS among four groups: 1) age 250 , HR-negative; 2) age 250 , HR-positive; 3) age <50, HR-negative; and 4) age <50, HRpositive using the full model described above (data not shown). Among all patient groups, BCT was consistently associated with improved OS when compared with mastectomy. The group achieving greatest benefit in overall survival with BCT relative to mastectomy were women age 50 and older at diagnosis with HR-positive tumors (HR 0.81, 95\% CI 0.790.84 ). The smallest benefit was seen among those women younger than age 50 at diagnosis with HR-positive tumors (HR 0.93, 95\% CI 0.86-0.99). Furthermore, the greatest benefit of BCT for DSS was seen among women age 50 and older at diagnosis with HR-positive disease (HR 0.87, 95\% CI 0.82-0.92), with other groups showing minimal or no significant benefit of BCT over mastectomy (Table 2).

Kaplan-Meier survival estimates showed significantly greater OS and breast cancer-specific survival favoring BCT over mastectomy. This benefit was seen in women in younger and older age groups, as well as those with HR-positive and HR-negative disease (Table 2, Figures 2A, 2B). The interaction between tumor size and surgical treatment was further evaluated. The OS and DSS benefit of BCT were greater among T1 than T2 tumors in all age and HR status strata analyzed. However, OS favored the BCT group even among those with $\mathrm{T} 2$ tumors. The advantage favoring BCT was greatest in women age 50 and older at diagnosis with HR-positive disease (HR 0.86, 95\% CI 0.82-0.91).

In order to estimate whether greater burden of underlying comorbidities could have impacted treatment choice by BCT or mastectomy, several different competing causes of mortality were evaluated. We examined several outcomes independently including OS, DSS, heart disease-specific survival, other cancer-specific survival, cerebrovascular diseasespecific survival and respiratory disease-specific survival. To more specifically evaluate those comorbidities likely to have been present at the time of breast cancer diagnosis, we examined mortality by truncating follow-up at 3 years after diagnosis (Table 3 ). Notably, BCT was associated with significantly lower 3 -year mortality rates from all causes, with the lowest hazard seen for heart disease-specific survival (HR 0.51, 95\% CI 0.44-0.60) and respiratory disease-specific survival (HR $0.46,95 \%$ CI $0.34-0.62$ ). The smallest reduction in hazard favoring BCT was seen for breast cancer-specific survival (HR 0.85, 95\% CI 0.780.91); however, BCT was associated with improved survival for all outcomes. 


\section{DISCUSSION}

Numerous prospective randomized trials suggest that BCT and mastectomy result in equivalent overall survival. Despite this, recent studies have shown an increased rate of mastectomy for patient subgroups including younger women with early stage tumors, many of which would have presumably been amenable to BCT. One likely contributing factor leading to an increased uptake of mastectomy is a perception of worse outcomes among women receiving BCT for tumors with unfavorable factors (e.g. young age, ER-negative disease, Her2-positive disease) ${ }^{12}$. The goal of this study was to determine whether the equivalent outcomes from BCT and M predicted from RCTs were achieved in the general population, and to find whether there existed a subgroup for which this equivalence was not demonstrated. We observed the unanticipated finding that patients treated with mastectomy had a significantly lower overall survival and breast cancer-specific survival than women who underwent BCT, even after adjusting for tumor grade, proportion of positive nodes, race, SES, tumor size, age at diagnosis, and year of diagnosis.

Our findings have important implications for understanding the overall benefit of BCT at the population level. Long-term breast cancer-specific survival after BCT was either equivalent or superior to mastectomy in all age groups and HR tumor types, with almost 10 years of median follow up. The strongest association with outcome was observed in those women older than 50 with HR-positive tumors who had a $13 \%$ lower breast cancer mortality and $19 \%$ lower overall mortality with BCT than with mastectomy (Table 2). These findings support the notion that BCT when combined with radiation confers at least equivalent, and perhaps even superior survival to mastectomy as definitive breast cancer treatment, with the greatest potential benefit to be gained among postmenopausal women with ER-positive T1 tumors, but at least equivalent outcomes seen among all groups on multivariable analysis, with the exception of women with tumor size $4-5 \mathrm{~cm}$.

\section{Determinants of Locoregional Treatment}

To put these findings into context, it is important to consider the factors determining receipt of BCT or mastectomy, including both patient- and disease-related variables. We noted that non-Hispanic whites and women residing in higher SES neighborhoods were more likely to undergo lumpectomy between 1990 and 2004. Although we adjusted for age, race, and SES, there are additional patient-level variables that we could not account for in our model. These include health care access issues such as health insurance coverage, distance to closest radiation facility, availability of reconstructive surgeons, and provider-related factors, all of which have been shown to impact rates of $\mathrm{BCT}^{1,2,13-17}$. Further, the cancer registry does not collect information regarding individual health history such as comorbid conditions, previous chest wall radiation, tumor-to-breast ratio, and other medical contraindications to radiation or lumpectomy. In aggregate, it is likely that these unmeasured variables could have resulted in higher mastectomy rates in those patients with more limited access to medical care, biasing our findings towards higher overall mortality and specific-cause mortality among women undergoing mastectomy. The magnitude and consequence of these potential biases is difficult to estimate but may in part account for the observed findings.

Among the disease-related factors that were collected, larger tumor size was associated with greater likelihood of undergoing mastectomy as expected. However, other adverse tumor features which would not be expected to impact choice of breast surgery including positive node status and higher tumor grade also increased the likelihood of mastectomy. In addition, factors such as node status are commonly not known at the time that surgery is planned; nonetheless, $44 \%$ of women with node positive disease underwent BCT compared to 59\% with node-negative cancer. Clearly, this difference can be partly attributed to the larger tumor size in the mastectomy group. Recognizing this potential confounder, the final model 
was adjusted for tumor size as a continuous variable as well as for proportion of positive nodes, as the latter is a more precise measure of nodal tumor burden than node status as a binomial variable. Other pathologic variables such as lymphovascular invasion and extranodal extension, both measures of tumor aggressiveness, were not accounted for, nor was Her2neu status as these data were not routinely reported to cancer registries prior to 2004. Although we were not able to adjust for these factors, it is unlikely that their cumulative impact would override the influence of tumor size, node status, and tumor grade.

\section{Alternate explanations}

Many patient or tumor characteristics not reported to the cancer registry may have influenced the recommendation for and choice of lumpectomy or mastectomy. One important source of confounding could have been competing causes of mortality from concurrent comorbidities, which could not directly be assessed with the California Cancer Registry data. Indeed, Giordano et al. cautions against misinterpreting treatment and mortality effects based on population-based cancer registry data, due to concerns about confounding by indication ${ }^{18}$. However, we conducted several analyses to directly address these concerns, including evaluating mortality due to varying causes of death within 3-years of the index breast cancer diagnosis as a proxy for preexisting comorbidities which may have influenced surgical treatment decisions. Interestingly, for every cause of mortality that we evaluated, women who had mastectomy were more likely to die within 3 years of their breast cancer diagnosis than women who chose BCT. Based on these findings, it is reasonable to infer that the mastectomy group was likely to have a greater burden of nonfatal comorbidities at presentation, and that this factor may well have influenced surgical decision-making. Nevertheless, this factor alone cannot account for why women with mastectomy had lower DSS after adjusting for age and tumor characteristics.

\section{Strengths and limitations of study design}

The California Cancer Registry is a large and representative source of long-term outcome data for women diagnosed and treated for breast cancer within the state of California. The strength of such a data source is that it allows a greater understanding of how BCT and mastectomy are used in a general and ethnically diverse population, not specific to particular health care institutions or settings. The long duration of follow-up provided in this study is important in order to minimize over-representation of breast cancer mortality by those tumor subtypes known to recur early.

There are clearly important limitations inherent in using a large population-based dataset. As evidenced above, cancer registries such as the CCR are not equipped to collect granular clinical data such as coexisting comorbidities, detailed pathologic data, and individual patient and provider biases, all of which may impact choice of treatment. Cancer registry data on radiation therapy are known to be under-captured, due to radiation that is administration in free-standing facilities, which is not always reported to the regional cancer registry. ${ }^{19,20}$. However, none of these factors would be expected to result in a sufficiently strong bias to override the known impact of tumor size, node status, age at diagnosis, race, and SES, all of which were included in the final multivariable model.

\section{Conclusion}

In a population-based cohort with early stage breast cancer, BCT was independently associated with an advantage in breast cancer specific survival at almost 10 years of follow up. The magnitude of this benefit was greatest among women 50 years or older at diagnosis with HR-positive tumors, although this effect was seen regardless of HR status and age at 
diagnosis. These results provide confidence in the efficacy of BCT even among younger patients with HR-negative disease thought to be at relatively higher risk for local failure.

\section{Acknowledgments}

The authors thank Dr. David O. Nelson for his help with this manuscript. This research was supported by the National Cancer Institute's Surveillance, Epidemiology and End Results Program under contract HHSN261201000140C awarded to the Cancer Prevention Institute of California. The collection of cancer incidence data used in this study was supported by the California Department of Health Services as part of the statewide cancer reporting program mandated by California Health and Safety Code Section 103885.

Funding Sources: none

\section{References}

1. Albain KS, Green SR, Lichter AS, et al. Influence of patient characteristics, socioeconomic factors, geography, and systemic risk on the use of breast-sparing treatment in women enrolled in adjuvant breast cancer studies: an analysis of two intergroup trials. J Clin Oncol. 1996; 14(11):3009-3017. [PubMed: 8918499]

2. Nattinger AB, Gottlieb MS, Veum J, Yahnke D, Goodwin JS. Geographic variation in the use of breast-conserving treatment for breast cancer. N Engl J Med. 1992; 326(17):1102-1107. [PubMed: 1552911]

3. Gilligan MA, Kneusel RT, Hoffmann RG, Greer AL, Nattinger AB. Persistent differences in sociodemographic determinants of breast conserving treatment despite overall increased adoption. Med Care. 2002; 40(3):181-189. [PubMed: 11880791]

4. Freedman RA, He Y, Winer EP, Keating NL. Trends in racial and age disparities in definitive local therapy of early-stage breast cancer. J Clin Oncol. 2009; 27(5):713-719. [PubMed: 19103731]

5. Gross CP, Smith BD, Wolf E, Andersen M. Racial disparities in cancer therapy: did the gap narrow between 1992 and 2002? Cancer. 2008; 112(4):900-908. [PubMed: 18181101]

6. Gomez SL, Lichtensztajn D, Kurian AW, et al. Increasing mastectomy rates for early-stage breast cancer? Population-based trends from California. J Clin Oncol. 2010; 28(10):e155-e157. author reply e58. [PubMed: 20159812]

7. Itakura K, Lessing J, Sakata T, et al. The impact of preoperative magnetic resonance imaging on surgical treatment and outcomes for ductal carcinoma in situ. Clin Breast Cancer. 2011; 11(1):3338. [PubMed: 21421520]

8. Katipamula R, Degnim AC, Hoskin T, et al. Trends in mastectomy rates at the Mayo Clinic Rochester: effect of surgical year and preoperative magnetic resonance imaging. J Clin Oncol. 2009; 27(25):4082-4088. [PubMed: 19636020]

9. van der Sangen MJ, van de Wiel FM, Poortmans PM, et al. Are breast conservation and mastectomy equally effective in the treatment of young women with early breast cancer? Long-term results of a population-based cohort of 1,451 patients aged $</=40$ years. Breast Cancer Res Treat. 2011; 127(1):207-215. [PubMed: 20703938]

10. Jones HA, Antonini N, Hart AA, et al. Impact of pathological characteristics on local relapse after breast-conserving therapy: a subgroup analysis of the EORTC boost versus no boost trial. J Clin Oncol. 2009; 27(30):4939-4947. [PubMed: 19720914]

11. Yost K, Perkins C, Cohen R, Morris C, Wright W. Socioeconomic status and breast cancer incidence in California for different race/ethnic groups. Cancer Causes Control. 2001; 12(8):703711. [PubMed: 11562110]

12. Nguyen PL, Taghian AG, Katz MS, et al. Breast cancer subtype approximated by estrogen receptor, progesterone receptor, and HER-2 is associated with local and distant recurrence after breast-conserving therapy. J Clin Oncol. 2008; 26(14):2373-2378. [PubMed: 18413639]

13. Boscoe FP, Johnson CJ, Henry KA, et al. Geographic proximity to treatment for early stage breast cancer and likelihood of mastectomy. Breast. 2011; 20(4):324-328. [PubMed: 21440439] 
14. Smith GL, Shih YC, Xu Y, et al. Racial disparities in the use of radiotherapy after breastconserving surgery: a national Medicare study. Cancer. 2010; 116(3):734-741. [PubMed: 20014181]

15. Chagpar AB, Studts JL, Scoggins CR, et al. Factors associated with surgical options for breast carcinoma. Cancer. 2006; 106(7):1462-1466. [PubMed: 16470610]

16. Elward KS, Penberthy LT, Bear H, Swartz DM, Boudreau RM, Cook SS. Variation in the use of breast-conserving therapy for Medicare beneficiaries in Virginia: clinical, geographic, and hospital characteristics. Clin Perform Qual Health Care. 1998; 6(2):63-69. [PubMed: 10180123]

17. Morrow M, Jagsi R, Alderman AK, et al. Surgeon recommendations and receipt of mastectomy for treatment of breast cancer. JAMA. 2009; 302(14):1551-1556. [PubMed: 19826024]

18. Giordano SH, Kuo YF, Duan Z, Hortobagyi GN, Freeman J, Goodwin JS. Limits of observational data in determining outcomes from cancer therapy. Cancer. 2008; 112(11):2456-2466. [PubMed: 18428196]

19. Jagsi R, Abrahamse P, Hawley ST, Graff JJ, Hamilton AS, Katz SJ. Underascertainment of radiotherapy receipt in surveillance, epidemiology, and end results registry data. Cancer. 2011

20. Virnig BA, Warren JL, Cooper GS, Klabunde CN, Schussler N, Freeman J. Studying radiation therapy using SEER-Medicare-linked data. Med Care. 2002; 40(8 Suppl):49-54. IV. 


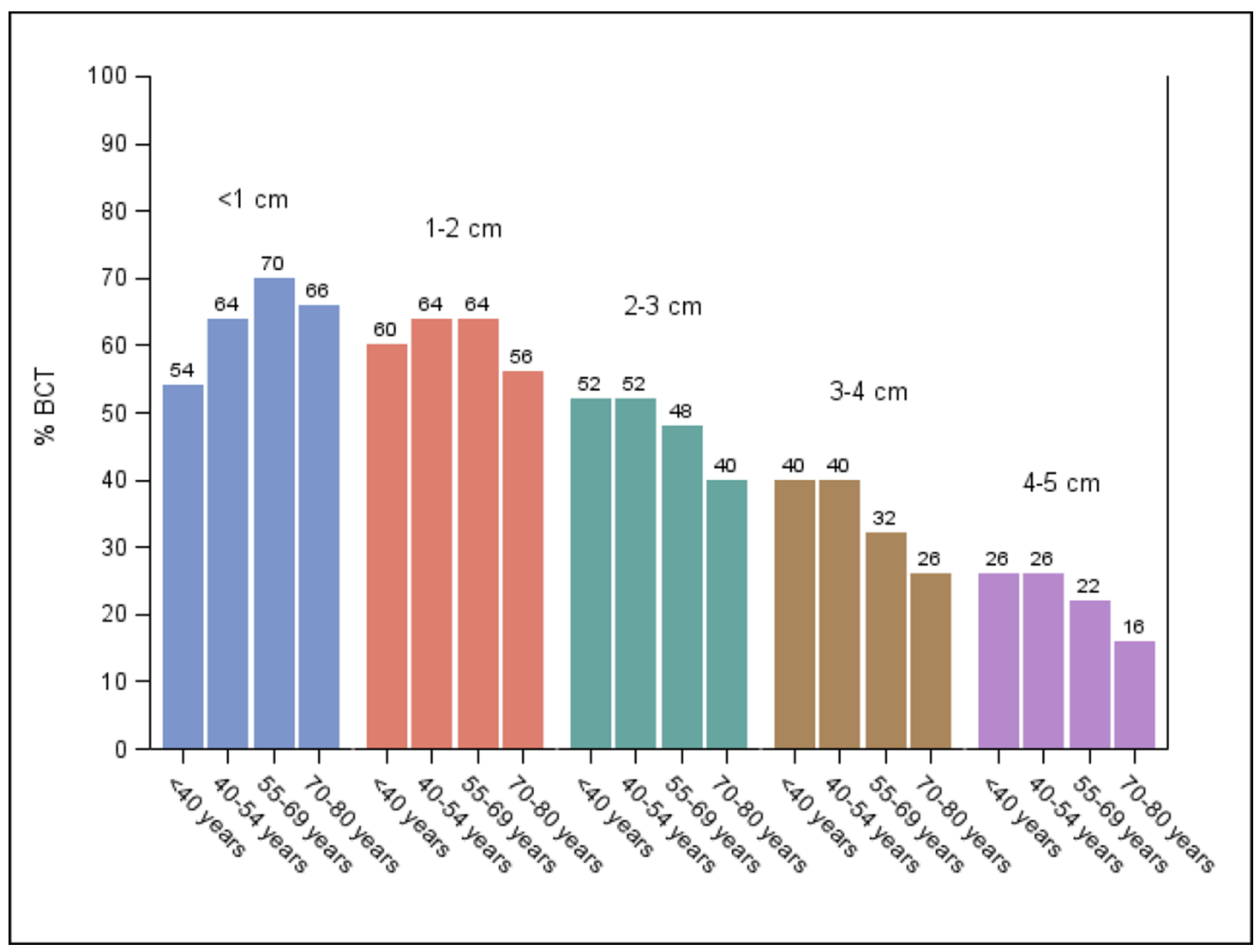

Figure 1.

The Impact of Tumor Size and Age at Diagnosis on rate of BCT in women with stage I or II breast cancer, 1990-2004 
A

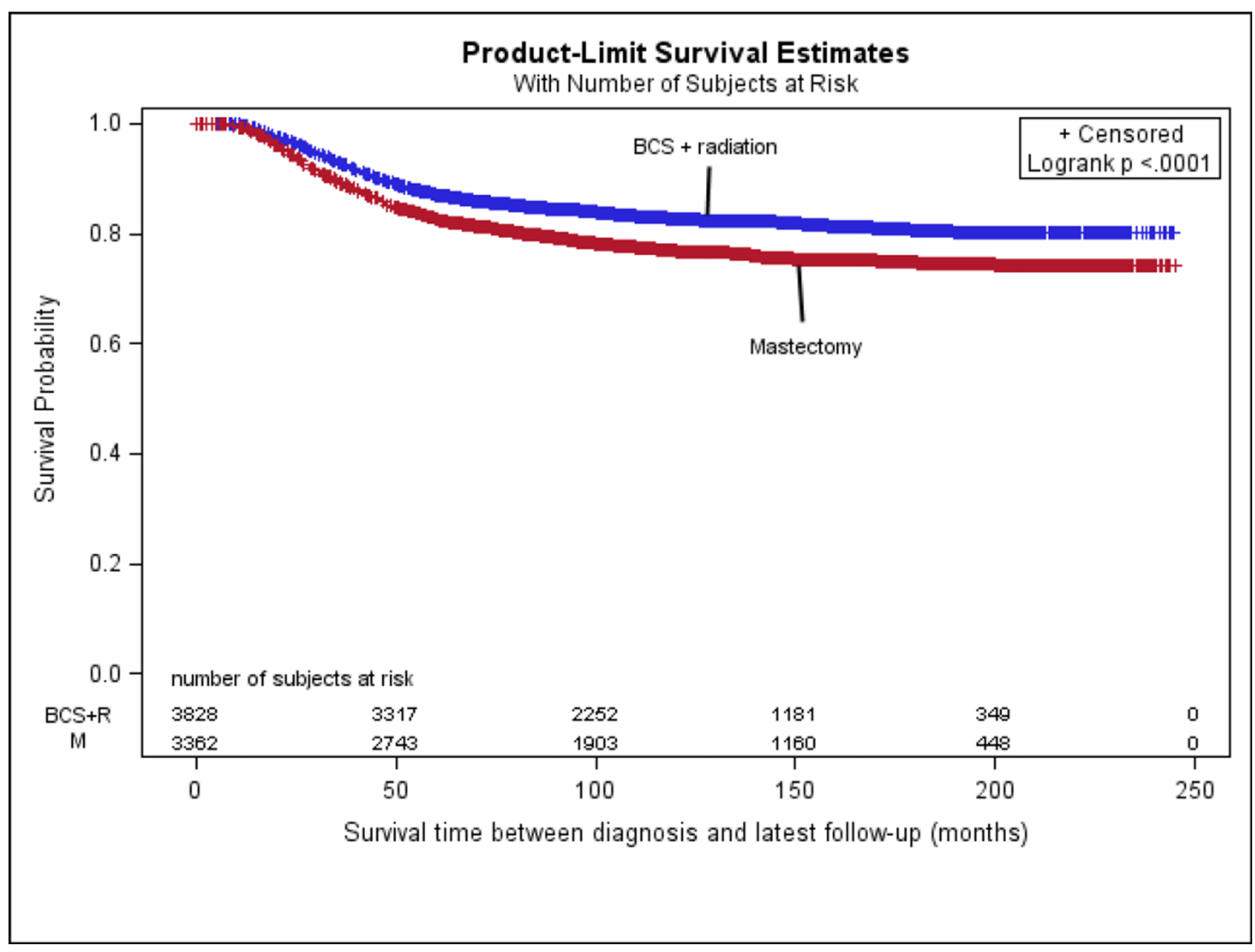


B

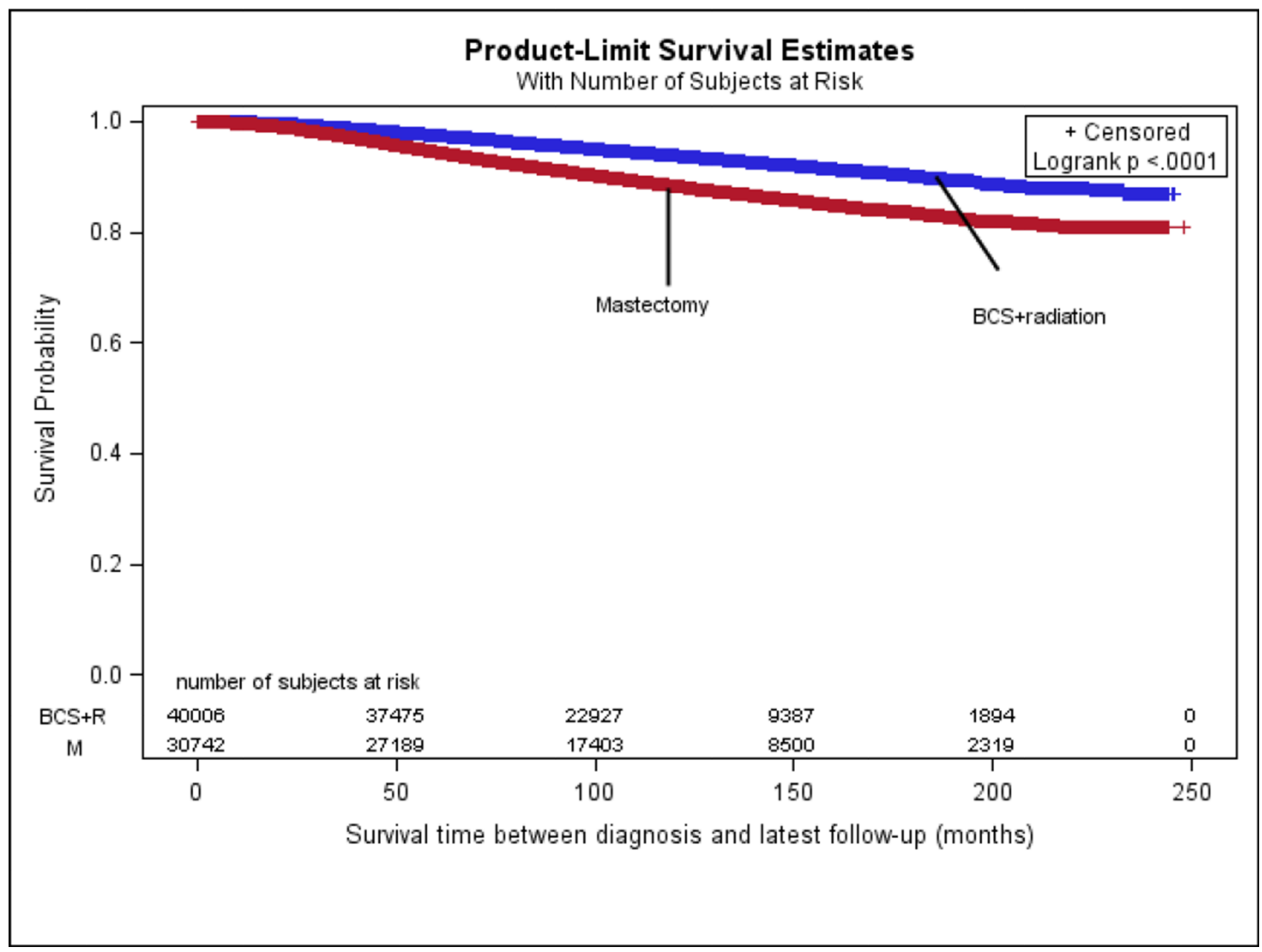

Figure 2.

A. Kaplan-Meier Overall Survival estimates for HR-negative patients, age $<50$ years at diagnosis: comparison of BCT and mastectomy groups.

B. Kaplan-Meier Overall Survival estimates for HR-positive patients, age $\geq 50$ years at diagnosis: comparison of BCT and mastectomy groups. 
Hwang et al.

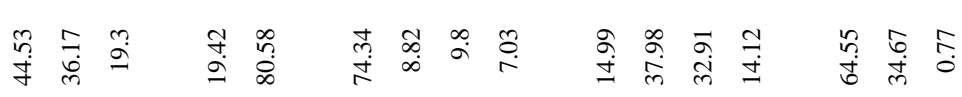

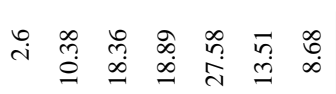

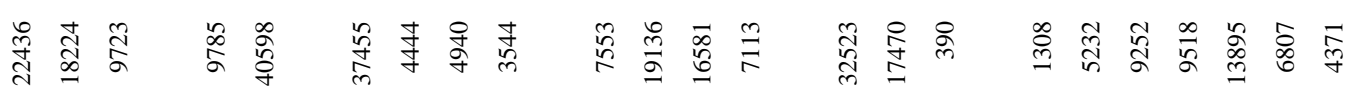

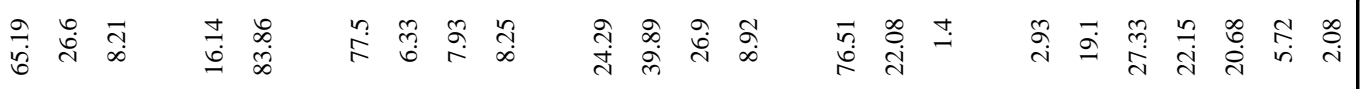

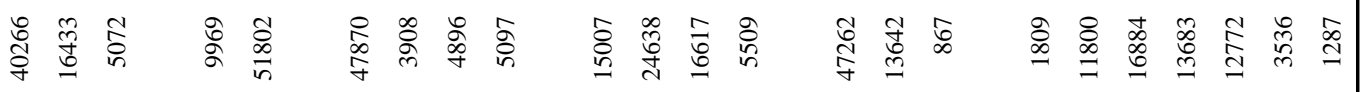




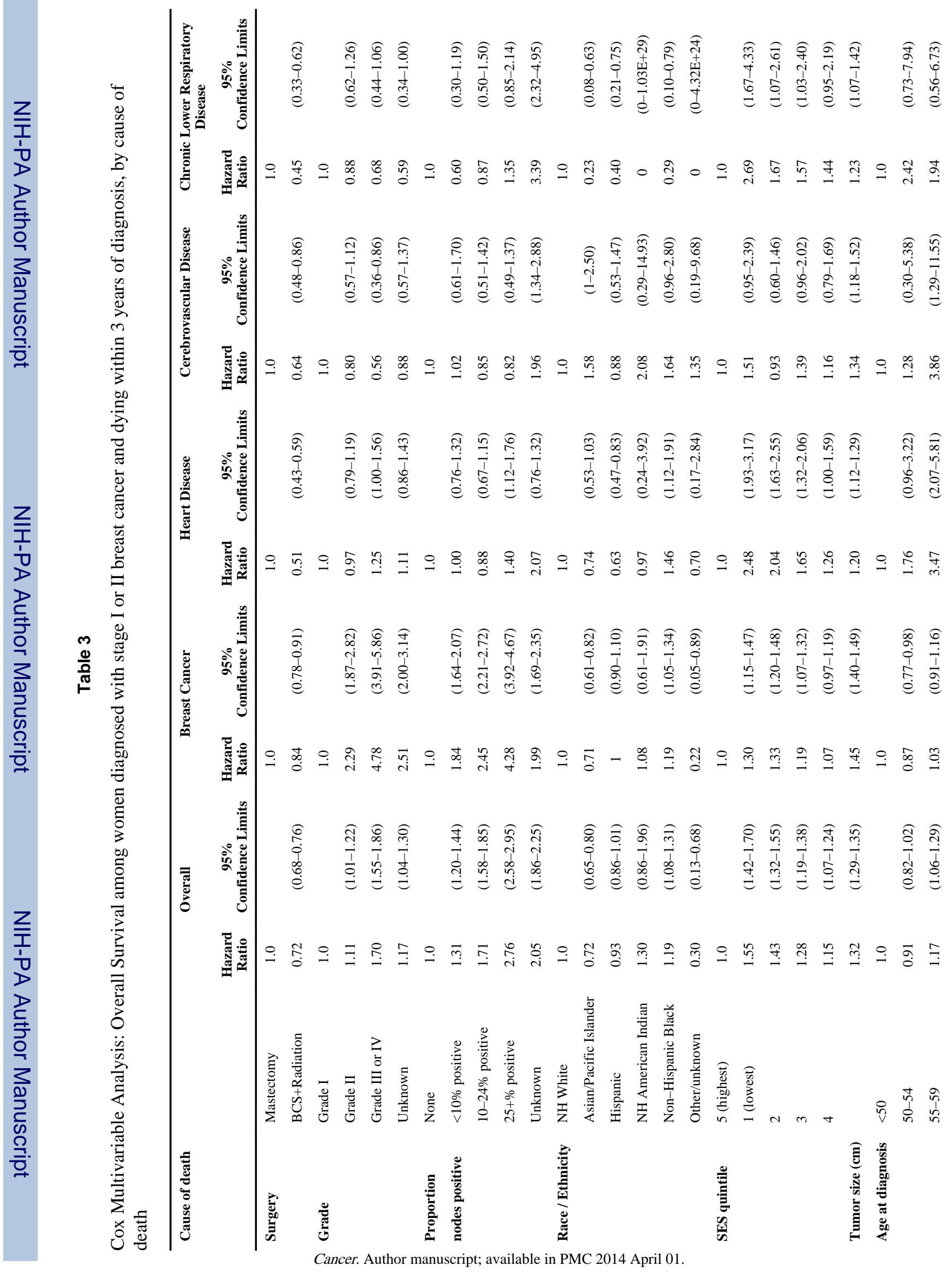




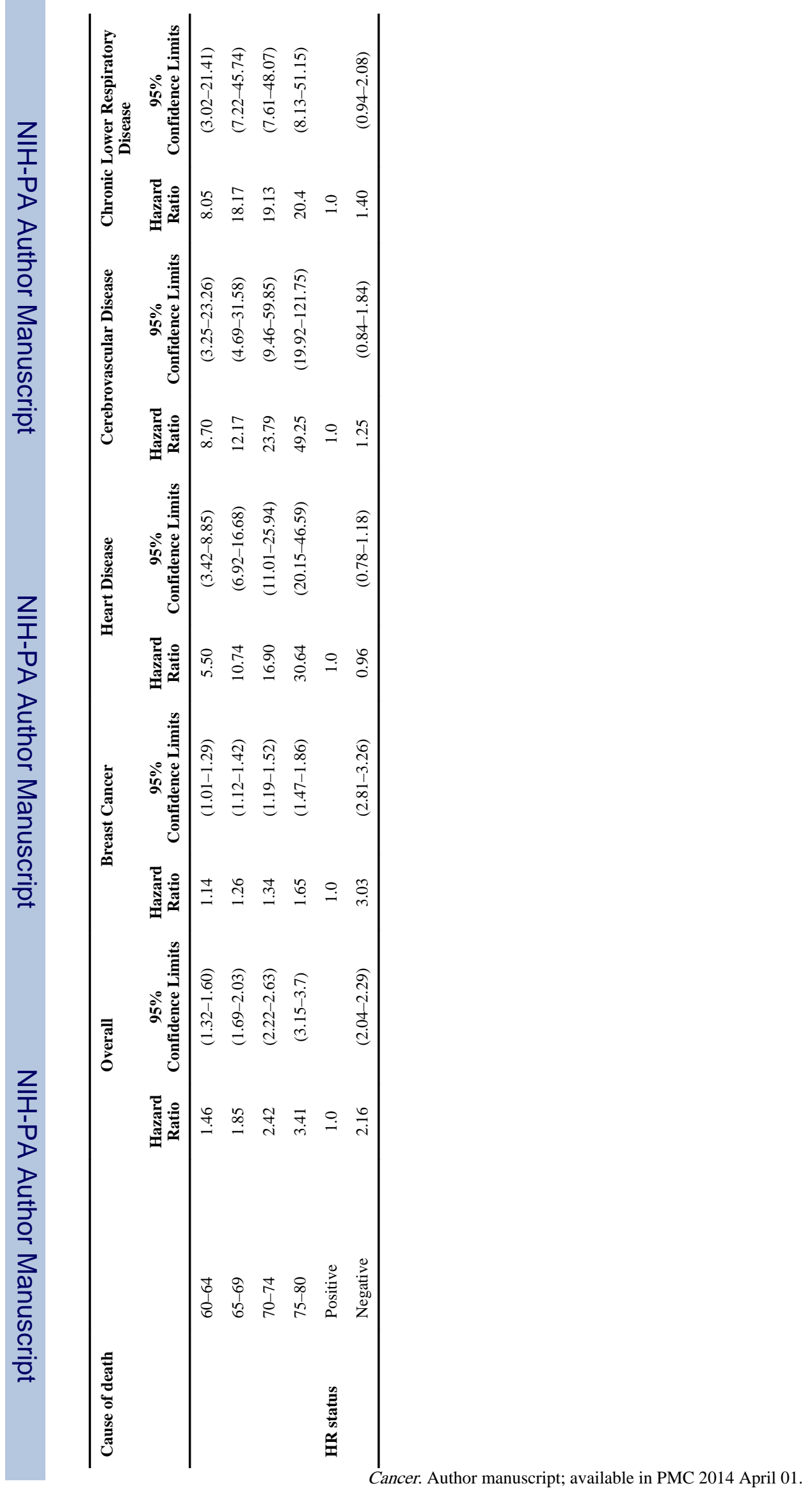

